\title{
Neutrino-photon reactions in astrophysics and cosmology
}

\author{
D. Seckel \\ Bartol Research Institute, University of Delaware, Newark DE 19716 \\ Bartol Preprint No. BA-97-32, Submitted to Phys. Rev. Lett.
}

(Sept 9, 1997)

\begin{abstract}
At energies above the threshold for $W$ production the process $\nu \gamma \rightarrow l W^{+}$is competive with $\nu \nu$ scattering at the same center of mass energies. In a cosmological setting, absorption of ultra high energy neutrinos by the microwave photon background is comparable to absorption by the neutrino background. In passing through matter, the process $\nu \rightarrow l W^{+}$will occur in the coulomb field of nuclei. For iron, the interaction rate per nucleon is roughly $20 \%$ of the charge current cross-section. The related process, $\bar{\nu}_{e} e^{-} \rightarrow \gamma W^{-}$dominates $\bar{\nu}_{e} e^{-}$scattering for about a decade in energy above the resonance for $W$ production.
\end{abstract}

13.10+q, 13.15+g, 95.30.Cq, 95.85.Ry, 98.70.Sa

Neutrinos of very high energy have become a subject of some interest [1]. Detection of such neutrinos could provide a means of identifying and studying sources of the highest energy cosmic rays [2, 3. 3 . Neutrinos, $\gamma$-rays, and nucleons are all produced at the source via hadronic reactions. Unlike photons or nucleons, however, neutrinos can both escape the central accelerator and propagate cosmological distances while preserving line of sight information to indicate the location of the source. High energy neutrinos from the decay or annihilation of particle dark matter [4] or from the decay of cosmic strings [5.6] could provide important clues for a deeper understanding of particle physics and/or cosmology. Recent models of $\gamma$-ray bursts may be testable by looking for coincident neutrinos with energies $E_{\nu}>10^{14} \mathrm{eV}$ [7].

With these thoughts in mind, experimental efforts are being initiated to detect cosmic neutrinos at energies from $10^{14}$ to $10^{20} \mathrm{eV}$ either underwater [8,9], underice 10,11, or possibly in horizontal air showers at an extensive air shower array [6]. Detection of such high energy neutrinos could be a boon for particle physicists as well. It is expected that such neutrinos would be absorbed by the Earth. By measuring the flux as a function of nadir angle one could measure neutrino-nucleon cross-sections at high energies [12]. Such a measurement would supply information about nucleon structure functions at energies inaccessible to current accelerators.

In all these cases, estimates of neutrino reaction rates have been based upon the exchange of weak vector bosons with nucleons or electrons, or in the case of cosmological absorption [13 16], the cosmic neutrino background. Here it is pointed out that neutrino-photon reactions that produce final state "on shell" weak vector bosons should not be neglected. The photon can be real, as in $\nu \gamma \rightarrow l W^{+}$, or virtual as in $\nu N \rightarrow N l W^{+}$catalyzed by the coulomb field of the nucleus. For the case of scattering from electrons, the photon may be in the final state, $\bar{\nu}_{e} e^{-} \rightarrow \gamma W^{-}$, which enhances $\bar{\nu}_{e} e^{-}$scattering above the "Glashow resonance" for $W$ production [17. These three cases are presented below followed by a summary and discussion.

$\nu \gamma \rightarrow l W^{+}$. It is straightforward to calculate the cross-section for $\nu \gamma \rightarrow l W^{+}$using the standard model lagrangian. A general form for the cross-section is

$$
d \sigma_{\nu \gamma \rightarrow l W^{+}}=|\mathcal{M}|^{2} \delta^{4}\left(p_{i}-p_{f}\right)(2 \pi)^{4} \frac{1}{4 I} d \rho_{f},
$$

where $p_{i}$ and $p_{f}$ denote initial and final particle fourmomenta, $\rho_{f}$ is the final particle phase space, $I$ is the lorentz invariant flux factor and $\mathcal{M}$ is the lorentz invariant amplitude for the process. Fig. (11) shows the two diagrams that contribute to $\mathcal{M}$. For the present purposes it is sufficient to consider the cross-section for unpolarized particles, so $|\mathcal{M}|^{2}$ may be simplified by summing

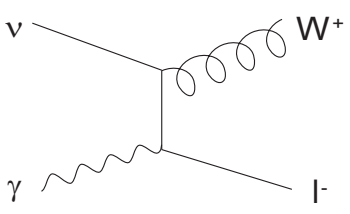

a)

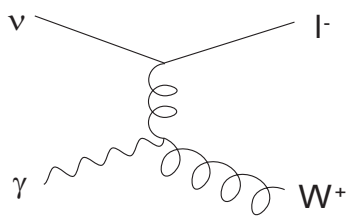

b)
FIG. 1. Two amplitudes contributing to $\nu \gamma \rightarrow l W^{+}:$a) is 'Compton like', while b) involves a three gauge coupling. Both must be included to maintain gauge invariance.

over $W$ polarizations, $\sum_{\lambda_{W}} \epsilon_{\lambda_{W}}^{\mu} \epsilon_{\lambda_{W}}^{\nu}=-\left(g^{\mu \nu}-\frac{p_{W}^{\mu} p_{W}^{\nu}}{M_{W}^{2}}\right)$. Before performing a similar sum over photon polarizations it is useful to calculate the electromagnetic current tensor $\mathcal{J}_{\mu \nu}=J_{\mu} J_{\nu}$, where $J$ is the current which couples to photons. The matrix element can then be written in the form $|\mathcal{M}|^{2}=\epsilon^{\mu} \epsilon^{\nu} \mathcal{J}_{\mu \nu}$, where $\epsilon$ is the photon polarization vector. For unpolarized photons one then uses the average $\frac{1}{2} \sum_{\lambda} \epsilon_{\lambda}^{\mu} \epsilon_{\lambda}^{\nu}=-\frac{1}{2} g^{\mu \nu}$. As a check of the algebra one can test that $p_{\gamma}^{\mu} \mathcal{J}_{\mu \nu}=p_{\gamma}^{\nu} \mathcal{J}_{\mu \nu}=0$ which is demanded by gauge invariance. $\mathcal{J}$ is also useful for calculating $\nu N \rightarrow N l W^{+}$in the nuclear coulomb field. 
Fig. (2) shows the cross-sections for $\nu \gamma \rightarrow l W^{+}$for the three different neutrino flavors. Near threshold, the lepton propagator in Fig. (19) leads to a large logarithm which enhances the cross-section for $\nu_{e}$ over that for $\nu_{\mu}$ and $\nu_{\tau}$. Setting the lepton mass to zero everywhere but in the logarithm, the cross-section is fairly compact:

$$
\begin{aligned}
\sigma_{\nu \gamma \rightarrow l W^{+}}= & \sqrt{2} \alpha G_{F}\left[2\left(1-\frac{1}{y}\right)\left(1+\frac{2}{y^{2}}-\frac{1}{y^{2}} \log y\right)+\right. \\
& \left.\frac{1}{y}\left(1-\frac{2}{y}+\frac{2}{y^{2}}\right) \log \frac{m_{W}^{2}(y-1)^{2}}{m_{e}^{2} y}\right], \quad
\end{aligned}
$$

where $y=s / m_{W}^{2}$ and $s=\left(p_{\nu}+p_{\gamma}\right)^{2}, G_{F}$ is Fermi's constant, and $\alpha$ is the fine structure constant which runs to $\sim 1 / 128$ near $m_{W}$.

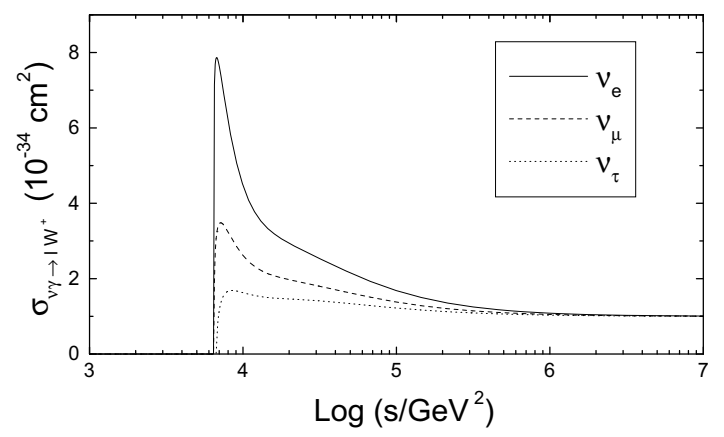

FIG. 2. Cross-section for $\nu \gamma \rightarrow l W^{+}$for three flavors of neutrino as a function of $s$ the squared center of mass energy. The threshold is at $s=\left(m_{W}+m_{l}\right)^{2}$.

It seems that the most interesting application of $\nu \gamma$ scattering is absorption of ultra high energy neutrinos off the microwave photon background. The potential importance of this process is illustrated in Fig. (3), where the $\nu_{e} \gamma$ cross-section is compared to relevant $\nu \nu$ and $\nu \bar{\nu}$ cross-sections at the same center of mass energies [14]. It is assumed that the neutrino mass is zero. The figure is dominated by processes involving intermediate $Z$ bosons at resonance, but at higher energies the $\nu \gamma$ cross-section is comparable or larger than that for the $\nu \nu$ reactions.

In a cosmological setting, the absorption rate is calculated by integrating the cross-section over the distribution of the target species. There are six flavors of neutrino and several processes to sum over. On the other hand photons have two spin degrees of freedom, and are more numerous than neutrinos by virtue of their higher temperature and boson statistics. Fig. (田) shows the absorption rate of high energy neutrinos from the cosmic background of photons or neutrinos. When $E_{\nu} \approx M_{Z}^{2} / T_{\nu}$ neutrino absorption is dominated by the $Z$ resonance, but at higher energies $\nu \gamma$ is important. At these higher energies the $\nu \nu$ processes mostly result in charged and neutral leptons, whereas the $\nu \gamma$ process produces $W^{+}$ bosons which mostly decay to quarks. Thus, not only is

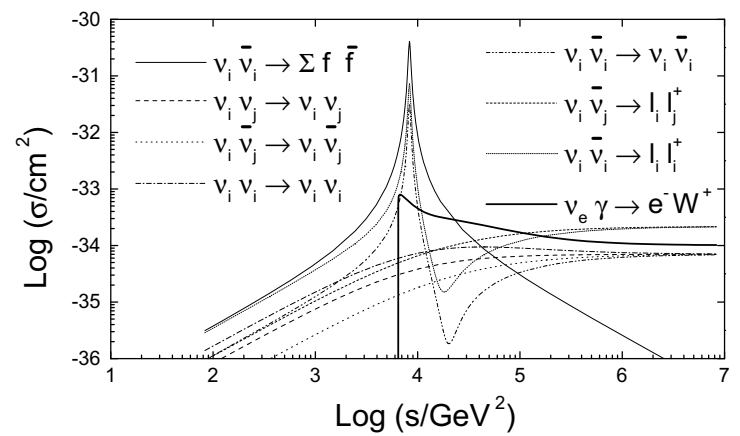

FIG. 3. Comparison of the $\nu_{e} \gamma$ cross-section to that for various $\nu \nu$ and $\nu \bar{\nu}$ processes as a function of $s$. The sum $\sum_{j} f_{j} \bar{f}_{j}$ does not include $f_{j}=\nu_{i}, l_{i}, t, W$, or $Z$.

the amplitude of the absorption modified, but also the character of the cascade products.

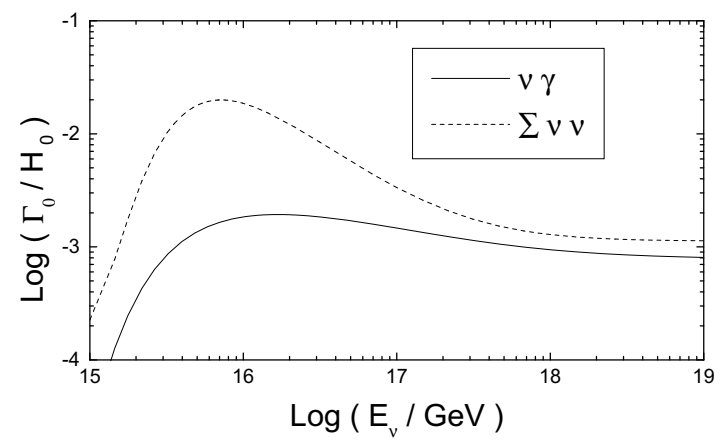

FIG. 4. Comparison of the absorption of high energy neutrinos from the cosmic background of photons and neutrinos to the cosmic expansion rate. The absorption by neutrinos includes a sum over the processes in Fig. (3). The photon temperature is taken to be $T_{\gamma}=2.74 \mathrm{~K}$, and $T_{\nu}=(4 / 11)^{1 / 3} T_{\gamma}$. The universe is assumed to be matter dominated with an expansion rate of $H_{0}=50 \mathrm{~km} / \mathrm{sec} / \mathrm{mpc}$.

In the present epoch these processes are important only for neutrinos with energies $E_{\nu}>10^{16} \mathrm{GeV}$, and even then only a small fraction of the beam is absorbed. Pushing back, neutrinos produced with energy $E_{\nu}(1+z)>10^{16} \mathrm{GeV}$ at redshifts $(1+z)>10$ would have been absorbed in their production epoch. A full cascade calculation must be done [16, evolving the ultra high energy neutrino distribution to low enough energies that they can propagate to the present unabsorbed. That cascade will be somewhat modified by the inclusion of $\nu \gamma$ reactions.

$\nu N \rightarrow N l W^{+}$. In addition to reactions with real photons, it is also possible to convert $\nu \rightarrow l W^{+}$in an external electromagnetic field. The most obvious case to consider is the coulomb field of a nucleus, where both significant field strength and momentum transfer are possible.

In the rest frame of the target nucleus, the cross-section 
per nucleon can be expressed as a convolution over scattering of the neutrino with the virtual photons in the coulomb field.

$$
d \sigma_{\nu N \rightarrow N l W^{+}}=d \sigma^{\prime} \frac{I^{\prime}}{I} \frac{Z^{2} e^{2} m_{N}^{2} F_{N}^{2}\left(q^{2}\right)}{A q^{4}} \frac{d^{3} q}{(2 \pi)^{3} 2 m_{N}}
$$

where $d \sigma^{\prime}$ is as in Eq. (11) except that the real photon is replaced by a virtual photon of momentum $q$ and polarization $j_{N}^{\mu} / m_{N}$. Here the electromagnetic current of the nucleus is defined as $e Z j_{N}^{\mu}$. In the rest frame of the nucleus, the matrix element used in $d \sigma^{\prime}$ is $\left|\mathcal{M}^{\prime}\right|^{2}=4 \mathcal{J}_{00}$, since in this frame $p_{N}^{\mu}=m_{N} \delta^{\mu 0}$ and we use $q^{\mu} J_{\mu}=0$. In Eq. (3) the quantity $I$ refers to the $\nu N$ system and $I^{\prime}$ refers to the $\nu$-virtual $\gamma$ system, so that $I^{\prime} / I=q z / m_{N}$, where $z$ is the direction cosine between the incident neutrino and $q ; Z$ and $A$ are the charge and atomic number of the nucleus; and $F_{N}$ is the form factor of the nucleus normalized to $F_{N}(0)=1$

$\mathcal{J}_{00}$ can then be expanded in powers of $q^{2} / m_{W}^{2}$, taking care to keep terms of order $E_{\nu}^{2} q^{2} / m_{W}^{4}$ until after the $d^{3} q$ integration is done. In this expansion, $m_{e}$ may be safely set to zero as the logarithm associated with the intermediate lepton is cut off by $q^{2}$ which is generally larger than $m_{e}^{2}$. For $\nu_{\mu}$ and $\nu_{\tau}$ conversion, the lepton mass should be kept. The highest momentum components of the nuclear field establish the threshold for conversion. These have momenta of roughly $100 \mathrm{MeV}$, so that $\nu N \rightarrow N l W^{+}$has a threshold of $E_{\nu} \approx 10^{14} \mathrm{eV}$. This is an interesting range for current and proposed underwater/ice neutrino detectors. In Fig. (5) we show the ratio of the cross-section per nucleon for $\nu_{e} N \rightarrow \mathrm{NeW}^{+}$, to that for charged current interactions [12] for the cases where the nuclear target is oxygen and iron, as a function of neutrino energy. The case of oxygen is interesting

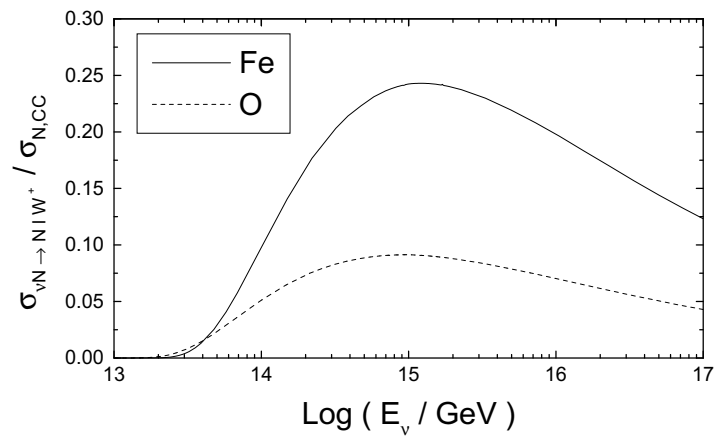

FIG. 5. Ratio of $\sigma_{\nu_{e} N \rightarrow N e W^{+}}$to that for $\sigma_{\nu N, c c}$. The cross-sections are per nucleon.

for neutrino detection rates in water (or ice) which are seen to increase by some $10 \%$ at $E_{\nu} \approx 1$ Pev. The crosssections on iron are increased by $20-25 \%$, which will have an impact on studies of nucleon structure functions based on absorption of high energy cosmic neutrinos by the Earth. At higher energies, the charged current crosssection increases roughly as $E_{\nu}^{0.4} 12$ whereas the photon exchange process increases only logarithmically and becomes less important.

$\bar{\nu}_{e} e^{-} \rightarrow \gamma W^{-}$. Neutrino interactions in matter are usually dominated by scattering with nucleons. An exception is the case of $\bar{\nu}_{e}$ : the $s$-channel reaction $\bar{\nu}_{e} e^{-} \rightarrow$ $W^{-} \rightarrow f \bar{f}^{\prime}$ is important near the $W$ resonance, although it decreases in importance at higher energy. Instead of the reaction with final state fermions $f \bar{f}^{\prime}$, it is also possible to produce on-shell $W$ 's accompanied by photons, $\bar{\nu}_{e} e^{-} \rightarrow \gamma W^{-}$, which is just the cross-channel of the $\nu_{e} \gamma \rightarrow e^{-} W^{+}$reaction considered above. As long as one does not work too close to the resonance, the crosssection involves only the two diagrams related to those in Fig. (11). Dropping $m_{e}$ except in the logarithm, the result is

$$
\begin{aligned}
\sigma_{\bar{\nu}_{e} e^{-}} & \rightarrow \gamma W^{-}=\frac{\sqrt{2} \alpha G_{F}}{3(y-1) y^{2}} \times \\
& \times\left[3\left(y^{2}+1\right) \log \left(\frac{y m_{W}^{2}}{m_{e}^{2}}\right)-\left(5 y^{2}+2 y+5\right)\right],
\end{aligned}
$$

where $y=s / m_{W}^{2}$ and here $s=2 m_{e} E_{\nu}$.

One might expect that with but a single channel and the smaller electromagnetic coupling that the $\gamma W^{-}$reaction would be less important than $f \bar{f}^{\prime}$ which proceeds to nine final states (12 above the top threshold). For very forward scattering, however, the $\bar{\nu}_{e} e^{-} \rightarrow \gamma W^{-}$process involves the $t$-channel exchange of an almost on-shell electron, which leads to an enhancement by $\log s / m_{e}^{2} \approx 25$. As a result the $\gamma W^{-}$rate exceeds the $s$-channel rate to $f \bar{f}^{\prime}$ summed over all species, as can be seen in Fig. (6); i.e. the cross-section for $\bar{\nu}_{e} e^{-} \rightarrow \gamma f \bar{f}^{\prime}$ exceeds that for $\bar{\nu}_{e} e^{-} \rightarrow f \bar{f}^{\prime}$.

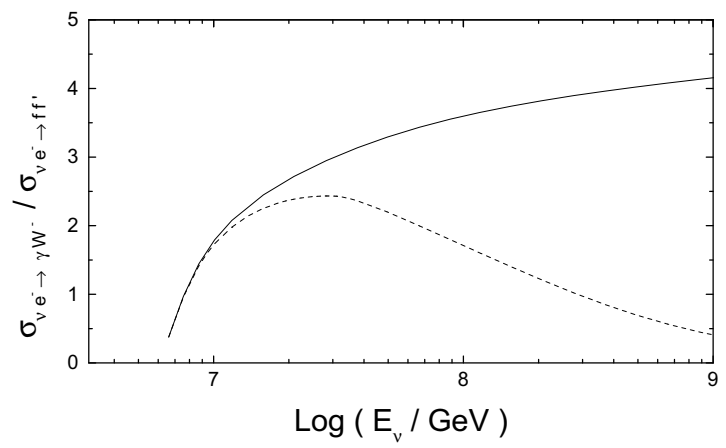

FIG. 6. Ratio of the cross-section for $\bar{\nu}_{e} e^{-} \rightarrow \gamma W^{-}$to that for $\bar{\nu}_{e} e^{-} \rightarrow f \bar{f}^{\prime}$. For the solid curve the sum over $f \bar{f}^{\prime}$ includes only the $s$-channel to final states open in $W$ decay. The dashed curve includes the $b \bar{t}$ final state as well as the $t$-channel $Z$ exchange for elastic scattering.

At high energies, $t$-channel $Z$-boson exchange allows the elastic channel to dominate so the importance of 
$\gamma W^{-}$decreases. For energies within a decay width of the resonance, a simple separation of the two processes is not possible - the photon is soft and so interference with initial and final state bremstrahlung emission must be considered [18]. For energies outside the width of the resonance, the photon produced in $\bar{\nu}_{e} e^{-} \rightarrow \gamma W^{-}$is hard and so will not interfere with bremstrahlung.

Summary. Neutrinos are generally considered to be weakly interacting particles, and thus neutrino-photon interactions are generally ignored, or confined to discussions of loop effects in scattering [19] or generating neutrino magnetic moments 20]. Here it is noted that for center of mass energies sufficient to produce real $W$ bosons that neutrinos and photons engage in $2 \rightarrow 2$ scattering at tree level via lepton exchange and through $\mathrm{W}$ exchange and a $\gamma W W$ vertex. The resultant $\nu \gamma$ reactions are competitive with traditional neutrino reactions at high energies and in some cases may be dominant.

Three examples have been explored: a) $\nu \gamma \rightarrow l W^{+}$is an important contribution to ultra high energy neutrino absorption in the early Universe, b) $\nu N \rightarrow N l W^{+}$catalyzed by the nuclear coulomb field enhances $\nu N$ reaction rates by $10-20 \%$ at neutrino energies of order $10^{15} \mathrm{eV}$, a range of interest to the next generation of neutrino telescopes, and c) the tree level process $\bar{\nu}_{e} e^{-} \rightarrow \gamma W^{-}$is the dominant reaction in $\bar{\nu}_{e} e^{-}$scattering for about a decade in $E_{\nu}$ just above the $W$ resonance.

The current discussion has been confined to real $W$ production, but it should be apparent that neutrinophoton processes below threshold may also occur. Below threshold, however, the virtual $W$ must decay, which results in two suppressions: the final state will have three particles instead of two, which reduces the available phase space by about a factor of 100; and the decay vertex adds two powers of the weak coupling constant which is a further reduction by about a factor of 10 . In a cosmological setting the process $\nu \gamma \rightarrow l f \bar{f}^{\prime}$ would therefore be expected to be about a factor of 1000 smaller than corresponding $\nu \nu$ scattering at similar center of mass energies.

More interesting is the possiblity of $\nu N \rightarrow N l f \bar{f}^{\prime}$ proceeding in the field of a nucleus. Even considering the $Z^{2}$ enhancement of the cross-section, the cross-section should be much reduced compared to the $\nu N$ charge exchange cross-section. However, for an experiment such as the detection of solar neutrinos at Kamiokande [21] the relevant comparison is to $\nu e$ elastic scattering which is also suppressed relative to $\nu N$ scattering so it is unclear if 'coulomb' scattering of neutrinos is unimportant. At slightly higher energies, even a modest contribution to $\nu N$ scattering in the few hundred $\mathrm{MeV}$ range could change interpretations of the atmospheric neutrino anomaly 22.

Acknowledgments. I am grateful to S. Barr, S. Pittel and T. Stanev for helpful suggestions and advice. This work was partially supported by DOE grant DE-FG0291ER40626.

[1] T.K. Gaisser, F. Halzen, and T. Stanev, Phys. Rep. 258, 173 (1995).

[2] F. Stecker, C. Done, M. Salamon, and P. Sommers, Phys. Rev. Lett. 66, 2697 (1991).

[3] A. Szabo and R. Protheroe, High Energy Neutrino Astrophysics, eds. V.J. Stenger, et al., World Scientific (Singapore) (1992) 24.

[4] Reviews: J. Primack, B. Sadoulet and D. Seckel, Ann. Rev. Nucl. Part. Sci. 38, 751 (1988); G. Jungman, M. Kamionkowski and K. Griest, Phys. Rep. 267, 195 (1996).

[5] J. Ostriker, G. Thompson and E. Witten, Phys. Lett. 180B, 231 (1986); C. Hill, D. Schramm and T. Walker, Phys. Rev. D36, 1007 (1987).

[6] J.J. Blanco-Pillado, R.A. Vazquez and E. Zas, Phys. Rev. Lett. 78, 3614 (1997); S. Yoshida, H. Dai, C.H. Jui and P. Sommers, Astrophys. J. 479, 547 (1997).

[7] E. Waxman and J. Bahcall, Phys. Rev. Lett. 78, 2292 (1997).

[8] BAIKAL Collaboration, The BAIKAL Neutrino Telescope NT-200, BAIKAL 92-03 (1992)

[9] Proceedings of the NESTOR Workshop at Pylos, Greece, ed. L.K. Resvanis, October 1992, U. Athens (1993)

[10] S. Barwick, et al. (AMANDA, Jour. Phys. G18, 225 (1992)

[11] C. Allen, et al., "Status of Radio Ice Cerenkov Experiment (RICE)", HE 4.1.26, Proc. XXV Int. Cosmic Ray Conf. Durban, (1997).

[12] R. Gandhi, C. Quigg, M.H. Reno, and I. Sarcevic, Astropart. Phys. 5, 81 (1996).

[13] V. Berezinsky, Nucl. Phys. B380, 478 (1992).

[14] E. Roulet, Phys. Rev. D47, 5247 (1993).

[15] P. Gondolo, G. Gelmini, and S. Sarkar, Nucl. Phys. B392, 111 (1993).

[16] S. Yoshida, Astropart. Phys. 2, 187 (1994).

[17] S. Glashow, Phys. Rev. 118, 360 (1960).

[18] D. Wackeroth and W. Hollik, Phys. Rev. D55, 6788 (1997)

[19] D.A. Dicus and W.W. Repko, Phys. Rev. Lett. 79, 569 (1997).

[20] Review: E.Kh. Akhmedov, hep-ph/9705451, 4th International Solar Neutrino Conference, Heidelberg, Germany, (1997).

[21] Z. Conner, et al., HE 4.1.22, Proc. XXV Int. Cosmic Ray Conf. Durban, (1997).

[22] T.K. Gaisser, Proc. Neutrino-96, Helsinki (1996). 